\title{
Effects of prolonged head-down bed rest on working memory
}

\author{
This article was published in the following Dove Press journal: \\ Neuropsychiatric Disease and Treatment \\ 26 March 2015 \\ Number of times this article has been viewed
}

\author{
Qing Liu',2 \\ Renlai Zhou ${ }^{1-4}$ \\ Xin Zhao ${ }^{5}$ \\ Tian Po S Oei ${ }^{6}$ \\ 'Beijing Key Lab of Applied \\ Experimental Psychology, School \\ of Psychology, ${ }^{2}$ Research Center \\ of Emotion Regulation, ${ }^{3}$ State \\ Key Laboratory of Cognitive \\ Neuroscience and Learning, \\ International Data Group/McGovern \\ Institute for Brain Research, Beijing \\ Normal University, Beijing, People's \\ Republic of China; ${ }^{4}$ Department \\ of Psychology, School of Social \\ and Behavioral Science, Nanjing \\ University, Nanjing, People's Republic \\ of China; ${ }^{5}$ Behavior Rehabilitation \\ Training Research Institution, School \\ of Psychology, Northwest Normal \\ University, Lanzhou, People's Republic \\ of China; 'School of Psychology, \\ University of Queensland, Brisbane, \\ QLD, Australia
}

Background: The weightlessness caused by prolonged bed rest results in changes in cerebral circulation and thus, brain functions, which is of interest.

Methods: We investigated the effects of 45 -day, $-6^{\circ}$ head-down bed rest, which stimulated microgravity, on working memory in 16 healthy male participants. The 2-back task was used to test the working memory variations on the 2 nd day before bed rest (R-2); on the 11th (R11), 20th (R20), 32nd (R32), and 40th (R40) days of bed rest; and on the eighth day after bed rest $(\mathrm{R}+8)$. The cognitive response and the physiological reactivity (such as galvanic skin response, heart rate, and heart rate variability) under the 2-back task were recorded simultaneously.

Results: The results showed that compared with $\mathrm{R}-2$, on the $\mathrm{R}+8$, the participants' galvanic skin response increased significantly, and the high frequency of heart rate variability (HF), low frequency of heart rate variability (LF), and reaction time in the 2-back task decreased significantly. There were positive correlations between the participants' reaction time of working memory and the LF/HF under head-down bed rest (at R11, R20, and R32).

Conclusion: The results suggested that the prolonged head-down bed rest may have a detrimental effect on individual physiology and working memory. Physiology indices, such as galvanic skin response and heart rate variability, were sensitive to the prolonged bed rest.

Keywords: galvanic skin response, heart rate, heart rate variability

\section{Introduction}

Outer space has an extreme living and working environment to which humans cannot naturally adapt and to which complex physiological and psychological adaptations are required. The extreme nature of space makes spaceflight a complicated high-risk project with high costs and requiring a large staff. Therefore, some space-based research domains remain in the early stages, while ground-based research has remained a more economic and safe way to investigate the effects of spaceflight on humans. ${ }^{1,2}$ The bed rest model of microgravity gives an alternative to space-based research for how weightlessness influences humans. This model, particularly as conducted in a head-down position, has been used to investigate physiological changes associated with spaceflight, including those occurring to the cardiovascular system, body fluid regulation, skeletal muscle, and bone.,

Prolonged weightlessness affects all the physical functions, which naturally includes the effects on the autonomic nervous system. ${ }^{6}$ The study of Aubert et al collected the heart rate (HR), blood pressure, and respiration rates from five astronauts while they were conducting a short-duration spaceflight. ${ }^{7}$ They found that microgravity influenced the participants' cardiovascular reactivity under a standard cognitive load task. Specifically, the mental arithmetic task induced the changes of vagal nerve activity for the astronauts, with an increase of sympathetic nervous activity and a decrease of
Correspondence: Renlai Zhou Department of Psychology, School of Social and Behavioral Sciences, Nanjing University, Nanjing, 210023, People's Republic of China Tel +8602589680960 ext 418 Email rlzhou@nju.edu.cn 
parasympathetic nervous activity. However, these changes were not observed after the spaceflight. The sample for this study was small $(\mathrm{N}=5)$. Thus there is a need to further explore this issue. Therefore, the present study looked at whether the mental stress task under bed rest would cause cardiovascular changes in participants similar to those of astronauts during spaceflight.

The effects of bed rest on cognition have been investigated, ${ }^{8}$ but research of the integration of cognition and physiology under bed rest has been rare and inconsistent. Ishizaki et al investigated the effects of a 16-day head-down bed rest (HDBR) on executive functions in 12 healthy male participants. ${ }^{17}$ They found that there were no significant differences in participants ${ }^{9}$ performances in the table-tapping test, the trial-making test, the pointing test, and the rockpaper-scissors test, before and after the 16-day HDBR. However, Lipnicki and Gunga, and Belavý and Felsenberg conducted a 60 -day, $-6^{\circ}$ HDBR to study the effects time (before, under, and after the HDBR) on executive functions (Iowa gambling task, working memory, and flanker tasks). ${ }^{8}$ The results of Lipnicki et al showed that the prolonged bed rest had a detrimental effect on individual's executive functions; performance was found to be significantly worse for the Iowa gambling task during bed rest than in other sessions, and less obviously bad effects were found on working memory and the flanker task. Similar to the study of Lipinicki et al, Seaton et al adopted the Spaceflight Cognitive Assessment Tool for windows (WinSCAT) to investigate the effects of 60 or 90 days of HDBR on cognition in 13 healthy participants. ${ }^{10}$ Their results showed that there were no significant changes on cognition before and after the prolonged bed rest.

There are many potential causes to which the inconsistent results of the aforementioned studies could be attributed. First, the time duration for bed rest was an important factor. A short-duration bed rest that lasts for 1 to 2 weeks, just like a short-duration spaceflight, would not cause changes of performance and mental state, especially of the higher cognitive functions, in participants/astronauts. ${ }^{28}$ However, a long-duration bed rest that lasts for more than 6 weeks, just like a long-duration spaceflight, would have more complicated effects on participants' (astronauts') performance and mental state. ${ }^{12}$ Second, there was a significant difference in the choice of testing tasks. Tasks that easily caused the practice effect would have a strengthened effect as time went on, while tasks that involved automatic processes would show detrimental effects of HDBR. ${ }^{13}$ Lastly, based on the neurovisceral integration model of Thayer et al higher cognition (relay on prefrontal functions) and cardiovascular vagal activity share a common neural control circuit. ${ }^{14}$ Stronger cardiovascular vagal activity would predict the better performance on executive function tasks; however, the cardiovascular vagal activity is enhanced by aerobic exercise, so it would decrease under bed rest because of physical inactivity. This means the prefrontal functions would be changed under HDBR and may be damaged. ${ }^{15}$ Actually, executive functions alternations occur in all ages of life, which made it an important issue for researchers. ${ }^{16-21}$

Above all, the current study aimed to explore the physiology and working memory changes (we also tested the performance on flanker task ${ }^{22}$ ) of participants under long-duration HDBR conditions. The 2-back task was used to measure working memory changes before, during, and after HDBR, and polygraph (MP150; BIOPAC Systems, Inc., Goleta, CA, USA) recordings of galvanic skin response (GSR), HR, and HR variability (HRV) were simultaneously performed with the participants performing the 2-back task. Both objective and subjective indices were used to reflect working memory and physiological variations. Our theoretical rationale led us to hypothesize that prolonged bed rest had a detrimental effect on individuals' physiology. Moreover, we hypothesized that the relationship between individual physiology and working memory under HDBR was of covariation. The research aimed at profoundly and systematically revealing variations of individuals' physiology and working memory under long-duration HDBR and clarifying the controversy regarding physiology and higher cognition in simulation environments of real outer space.

\section{Methods \\ Ethics}

All participants provided written informed consent to participate in the present experiment. Experimental procedures were approved by the Institutional Review Board of the State Key Laboratory of Cognitive Neurosciences and Learning of Beijing Normal University. The study was performed in accordance with the ethical guidelines of the Declaration of Helsinki.

All participants received 15,000 Yuan compensations for participating in the experiment.

\section{Participants}

The participants in the HDBR study were 16 young healthy males. The subjects volunteered to participate in the present study and were selected after a screening evaluation including a physical examination and psychological questionnaire investigations. Due to lack of physiological data, 
one participant was excluded from analysis. The final 15 participants had a mean age of 26.33 (standard deviation [SD] 4.13) years, with the mean height of 170.33 (SD 4.09) $\mathrm{cm}$ and mean weight of 62.20 (SD 5.56) kg. They reported no color blindness, and their vision and audition were within the normal range. The participants had an educational background of at least technical secondary school or above. All of the participants were right-handed and nonathletes.

\section{Materials}

Working memory 2-back task: The classical 2-back task was used to measure the participants' working memory. This task was preferable for its reliability and validity. ${ }^{23}$ The procedure was carried out using E-prime ${ }^{\circledR} 2.0$ software. The task was a spatial working memory (2-back) task. In this task, there was a cross in the center of the black screen, and a number would appear above, below, left or right of the cross. The range of numbers was 1 to 9, the script was "Times New Roman", the color was white, and the font size was 60 . The numbers and their order were random. Participants were required to ignore the verbal meaning and judge whether the position of the number was the same as the alternating one. In each trail, first presented the prompt ' $X$ ', then followed by a $1300 \mathrm{~ms}$ delay, and present the number (stimuli) for $200 \mathrm{~ms}$, after which the participants should to make a response within $2500 \mathrm{~ms}$. The intervals between trails were $4500 \mathrm{~ms}$. Half of the participants pressed " 1 " for a matched stimulus and " 3 " for an unmatched one. The other half of the participants pressed the opposite. The task consisted of 101 trials, divided into three blocks; of these, one block had 17 trials for practice and the other two blocks each had 42 trials. The order of the matched and unmatched stimulus was random. Participants did not need to respond for the first two trials. In each task, the percentage of matched stimuli and unmatched stimuli was $50 \%$. We tested the individuals six times across the experiment, using the same 2-back task. Reaction time outliers were filtered using a $<150$ and $>2,000$ ms cutoff, with subsequent removal of all reaction times (RTs) exceeding 3.0 SD from the mean.

The stimuli of the 2-back task displayed on a 14-inch screen with a $1,024 \times 768$ resolution and $85 \mathrm{~Hz}$ refreshing rate. There was a $60 \mathrm{~cm}$ distance between the participants and the screen. The visual angle was $90^{\circ}$.

\section{Procedure}

The $-6^{\circ}$ HDBR condition lasted 45 days. The measurements taken were: 2 days before the HDBR $(\mathrm{R}-2)$; on the 11th day (R11), 20th day (R20), 32nd day (R32), and 40th day (R40); and finally, 8 days after the HDBR $(\mathrm{R}+8)$. At the pretest ( $\mathrm{R}-2)$ and the posttest $(\mathrm{R}+8)$, participants were instructed to lie in a horizontal position to do the experiment tasks. During the HDBR, participants did everything, such as eating, washing, bathing, and urinating, while lying in bed; exceptions were made for passing excrement and weighing. The participants were allowed to watch TV, play games, or read. Additionally, they could freely connect with the outside world by telephone. There were six to eight people in each room, and the beds were separated by a mobile curtain. The temperature of the room was maintained between $23^{\circ} \mathrm{C}$ and $27^{\circ} \mathrm{C}$. Before HDBR, all the participants were given a balanced diet to stabilize their basal nutrition states. During and after HDBR, they were kept on the diet to avoid the effects of differences in nutrition. The participants' blood pressure, $\mathrm{HR}$, respiratory rate, temperature, hydration, urination, and general states were monitored during HDBR. The rest schedules were strictly controlled, ie, all participants were woken up at $6 \mathrm{am}$, ate breakfast from 6:30 to $7 \mathrm{am}$, and ate lunch from $11: 30$ to $12: 30 \mathrm{am}$. Afternoon nap was from 12:30 to $1: 30 \mathrm{pm}$, dinner was from 5:30 to $6: 30 \mathrm{pm}$, and the sleep period was from $10 \mathrm{pm}$ to $6 \mathrm{am}$ the next day. During the remaining time of the day, the participants were given experiments (such as the flanker task, prospective memory task, and working memory task). However, in this study, we only reported the results of the working memory task and exercises; the results of the other two tasks were previously published). ${ }^{2,21}$ In order to access the effect of HDBR on individuals, the participants answered a postsurvey about their feelings, asking them about their feelings under HDBR (eg, "Did you sleep well under HDBR" and "Did you suffer under HDBR"). Before the experiment tasks started, physiological equipment (MP150) was attached to the participants; soon after, the 2-back working memory task was conducted, during which physiological indices, including GSR and electrocardiograph (ECG), were recorded.

\section{Data collection and analysis}

Physiological activity was recorded by a polygraph (BIOPAC MP150). The physiological indices that we recorded were the GSR and ECG. The electrodes for the GSR were attached to the participants' left-hand index finger (for the positive electrode) and left-hand middle finger (negative electrode). The amplifier gain of the GSR was $5 \mu \mathrm{mho} / \mathrm{V}$, the high-pass filter was DC (DC, gives direct/absolute GSR readings from the participant), which means signals higher than DC were allowed to come in and the low-pass filter was $1 \mathrm{nHz}$ (which means signals lower than $1 \mathrm{HZ}$ were allowed to come in). The unit of GSR was $\mu$ mho, which was ten negative six squares of the conductance. 
ECG signal data are transmitted at a rate of $500 \mathrm{~Hz}$. Raw data from the pair are band-limited from $0.05 \mathrm{~Hz}$ to $35 \mathrm{~Hz}$. The amplifier gain was 500, the high-pass filter was $0.5 \mathrm{~Hz}$ (which means that the signals higher than $0.5 \mathrm{HZ}$ were allowed to get in), and the low-pass filter was $35 \mathrm{~Hz}$ (which means that the signals which lower than $35 \mathrm{HZ}$ were allowed to get in). The HR of each participant was obtained on the basis of the R-R interval, which was immediately extracted from the ECG signal. The unit of the HR was beats/minute. The ground (GND) was connected to the right lower limb, the VIN+ (positive electrode) was connected to the left lower limb, and the VIN- (negative electrode), which showed the electrode connections to the ECG for the lead measurements, was connected to the left upper limb (normal lead II).

SPSS 16.0 software was used to conduct repeated measures analysis of variance (RM-ANOVA), and the significance level was described with a two-tail alpha of $0.05(P<0.05)$. Partial eta squared $\left(\eta^{p 2}\right)$ was presented as the effect size for ANOVA effects, and Greenhouse-Geisser correction was used where appropriate. Data were expressed as mean \pm standard error of the mean (SEM). Acknowledge 4.1 software was used to extract and analyze the participants' GSR, HR, and HRV (including the high frequency of HRV [HF]; the low frequency of HRV [LF]; and the balance [LF/HF]). The frequency domain information of $\mathrm{HRV} \operatorname{HF}(0.15 \mathrm{~Hz} 0.40 \mathrm{~Hz})$, LF $(0.04 \mathrm{~Hz} \sim 0.15 \mathrm{~Hz})$, and the LF/HF were transformed by the R-R variability via fast Fourier transform (FFT). In order to eliminate the skewness distribution, we transformed the raw data of HF and LF to a normalized form, with units of nu. This was useful when evaluating the effects of different interventions in the same subject. ${ }^{25}$ Normalized units were obtained as follows:

$$
\begin{aligned}
\operatorname{LF} \text { norm }(\mathrm{nu})= & \left\{\mathrm{LF}\left(\mathrm{ms}^{2}\right) /\left[\text { total power }\left(\mathrm{ms}^{2}\right)-\right.\right. \\
& \left.\left.\operatorname{VLF}\left(\mathrm{ms}^{2}\right)\right]\right\} \times 100
\end{aligned}
$$

HF norm $(\mathrm{nu})=\left\{\mathrm{HF}\left(\mathrm{ms}^{2}\right) /\left[\right.\right.$ total power $\left(\mathrm{ms}^{2}\right)-$

$$
\left.\left.\operatorname{VLF}\left(\mathrm{ms}^{2}\right)\right]\right\} \times 100
$$

\section{Results}

\section{Changes of individual physiology under $-6^{\circ} \mathrm{HDBR}$}

Changes of galvanic skin response under $-6^{\circ} \mathrm{HDBR}$

The GSR was measured by repeated measures ANOVA at different time points (R-2, R11, R20, R32, R40, and R+8). The results demonstrated that the main effect of GSR was significant $\left(F_{[5,70]}=7.350, P<0.001, \eta^{2}=0.344\right)$. Further paired-sample $t$-test analysis revealed that compared with $\mathrm{R}-2$, the participants' GSR decreased significantly during the HDBR $(P=0.022$ [R20]; $P<0.001$ [R11]; $P<0.001$ [R32]; and $P<0.001$ [R40]) and after the $\operatorname{HDBR}(P=0.002$
$[\mathrm{R}+8])$. Compared with R20 $(P=0.047)$ and R32 $(P=0.001)$, the participants' GSR increased significantly at the end of the HDBR (R40). The specific tendency is shown in Figure 1. In addition, the postintervention survey of participants showed no suffering or sleep problems under the HDBR.

\section{Changes of individual heart rate and heart rate variability under $-6^{\circ} \mathrm{HDBR}$}

HR and HRV were measured by repeated measures ANOVA at different time points (R-2, R11, R20, R32, R40, and $\mathrm{R}+8)$. The data showed that the main effect of $\mathrm{HR}$ at different time points was not significant $\left(F_{[5,70]}=1.729, P=0.139\right.$, $\left.\eta^{2}=0.110\right)$. The results of HRV demonstrated that the main effects of HF $\left(F_{[5,70]}=4.619, P=0.001, \eta^{2}=0.248\right)$ and LF $\left(F_{[5,70]}=4.752, P=0.001, \eta^{2}=0.253\right)$ were significant at different time points. Further paired-sample $t$-test analysis revealed that compared with $\mathrm{R}-2$, the participants' $\mathrm{LF}$ decreased significantly at the end of the HDBR $(P=0.046$ [R40]); the LF $(P=0.005)$ and the HF $(P<0.001)$ continued to decrease after the HDBR $(\mathrm{R}+8)$. Compared with R11, the participants' HF ( $P=0.047$ [R32]; $P=0.001$ [R40]; and $P=0.011[\mathrm{R}+8])$ and LF $(P=0.048$ [R32]; $P=0.009$ [R40]; and $P=0.012[\mathrm{R}+8])$ decreased significantly during the HDBR and after the HDBR. However, compared with the R20, the participants' HF $(P=0.011)$ and LF $(P=0.013)$ increased significantly after the HDBR $(\mathrm{R}+8)$. The main effect of LF/HF was not significant at different time points $\left(F_{[5,70]}=0.170, P=0.973, \eta^{2}=0.012\right.$. The raw data of HR and HRV are shown in Table 1.

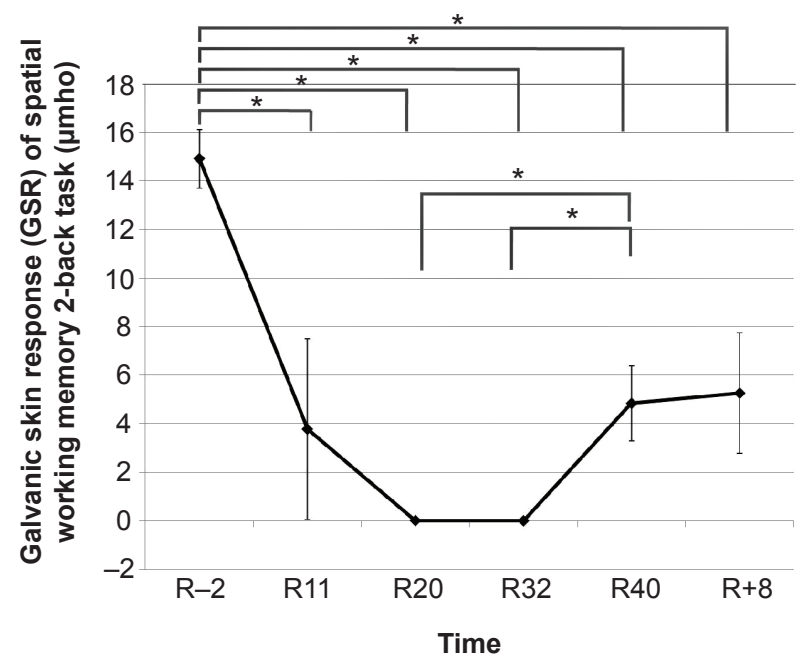

Figure I Participants' galvanic skin response of the spatial working memory 2-back task at different time points (R-2, RI I, R20, R32, R40, and R+8).

Notes: Time was expressed in milliseconds; $* P<0.05$.

Abbreviations: HDBR, head-down bed rest; R-2, 2 days before the HDBR; RII, day I I of HDBR; R20, day 20 of HDBR; R32, day 32 of HDBR; R40, day 40 of HDBR; $R+8,8$ days after the HDBR. 
Table I Changes in heart rate and heart rate variability (HF, LF, and LF/HF) of the participants at different test times (R-2, RII, R20, $\mathrm{R} 32, \mathrm{R} 40$, and $\mathrm{R}+8$ ), under the spatial working memory 2-back task (expressed as mean $\pm \mathrm{SE}$ )

\begin{tabular}{|c|c|c|c|c|c|c|}
\hline & \multicolumn{6}{|l|}{ Time points } \\
\hline & $\mathbf{R}-\mathbf{2}$ & RII & $\mathbf{R} 20$ & $\mathbf{R 3 2}$ & R40 & $\mathbf{R}+\mathbf{8}$ \\
\hline & $M(S E)$ & $M(S E)$ & $M(S E)$ & $M(S E)$ & $M(S E)$ & $M(S E)$ \\
\hline Heart rate (bpm) & $71.92(4.28)$ & $70.23(3.63)$ & $68.50(2.21)$ & $70.64(2.22)$ & $74.48(2.12)$ & $72.26(3.15)$ \\
\hline Low frequency (nu) & $45.47(0.34)$ & $45.48(0.50)$ & $45.60(0.25)$ & $45.53(0.17)$ & 45.7I (0.38) & $45.41(0.10)$ \\
\hline High frequency (nu) & $54.53(0.34)$ & $54.52(0.50)$ & $54.40(0.25)$ & $54.47(0.17)$ & $55.29(0.38)$ & $55.59(0.10)$ \\
\hline LF/HF & $0.84(0.01)$ & $0.84(0.02)$ & $0.84(0.01)$ & $0.84(0.01)$ & $0.84(0.01)$ & $0.83(0.01)$ \\
\hline
\end{tabular}

Note: The high-frequency and low-frequency data that are shown in table I were normalized and their units are 'nu'.

Abbreviations: HDBR, head-down bed rest; HF, high frequency of heart rate variability; LF, low frequency of heart rate variability; R-2, 2 days before the HDBR; RI I, day II of HDBR; R20, day 20 of HDBR; R32, day 32 of HDBR; R40, day 40 of HDBR; R+8, 8 days after the HDBR; SE, standard error of the mean.

\section{Changes of working memory under $-6^{\circ} \mathrm{HDBR}$}

The accuracy (ACC) and RT of working memory in the 2-back task were computed by repeated measures ANOVA at different time points (R-2, R11, R20, R32, R40, and R+8). It was found that the main effect of ACC was significant $\left(F_{[5,70]}=2.393, P=0.046, \eta^{2}=0.146\right)$. Further paired-sample $t$-test analysis showed that compared with $\mathrm{R}-2$, participants' ACC increased significantly during $\operatorname{HDBR}(P=0.008$ [R20]; $P=0.009$ [R32]; and $P=0.028$ [R40]), which is shown in Figure 2A. Additionally, the main effect of RT was also significant $\left(F_{[5,70]}=17.632, P<0.001, \eta^{2}=0.557\right)$. Further analysis demonstrated that compared with $\mathrm{R}-2$, the participants' RT decreased significantly during HDBR $(P<0.001$ [R11]; $P<0.001$ [R20]; $P<0.001$ [R32]; and $P<0.001$ [R40]) and at the $\mathrm{R}+8(P<0.001)$. Compared with R11, the participants' RT decreased significantly on R20 ( $P=0.037)$,
$\mathrm{R} 40(P=0.050)$, and $\mathrm{R}+8(P=0.038)$. The specific tendency is shown in Figure 2B.

\section{Correlations of physiological activities and} the performance of the 2-back task

Pearson correlations were conducted between the working memory 2-back task performance (ACC and RT) and the physiological data (GSR, HR, LF, HF, and LF/HF) recorded under the HDBR condition at different time points ( $\mathrm{R}-2$, R11, R20, R32, R40, and R+8). The results of the Pearson correlations are shown in Table 2.

The results showed that the participants' GSR and ACC of 2-back task had a significantly negative correlation on R11 ( $r=-0.528, P=0.043$ ), while the LF/HF and the RT of 2-back task had a significantly positive correlation on R11 ( $r=0.515$, $P=0.049$ ). The result indicated that at the beginning of the
A

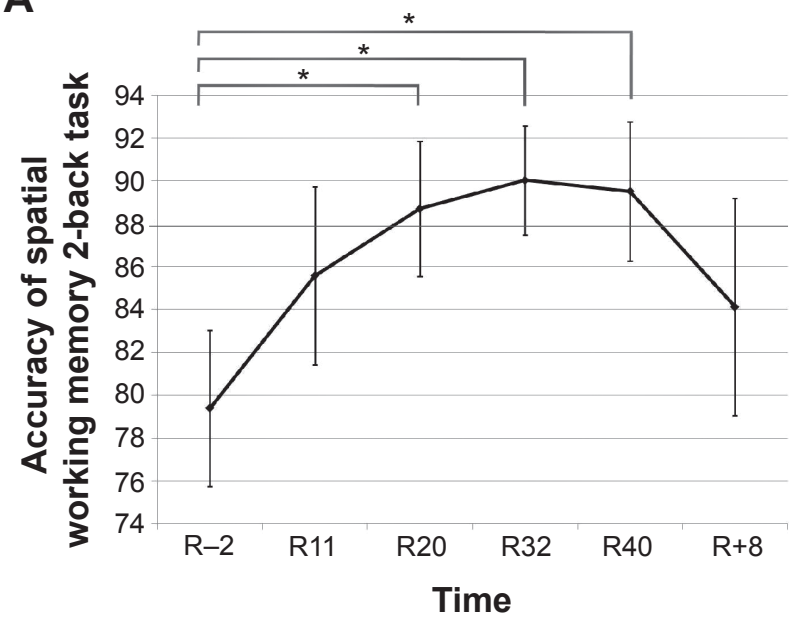

B

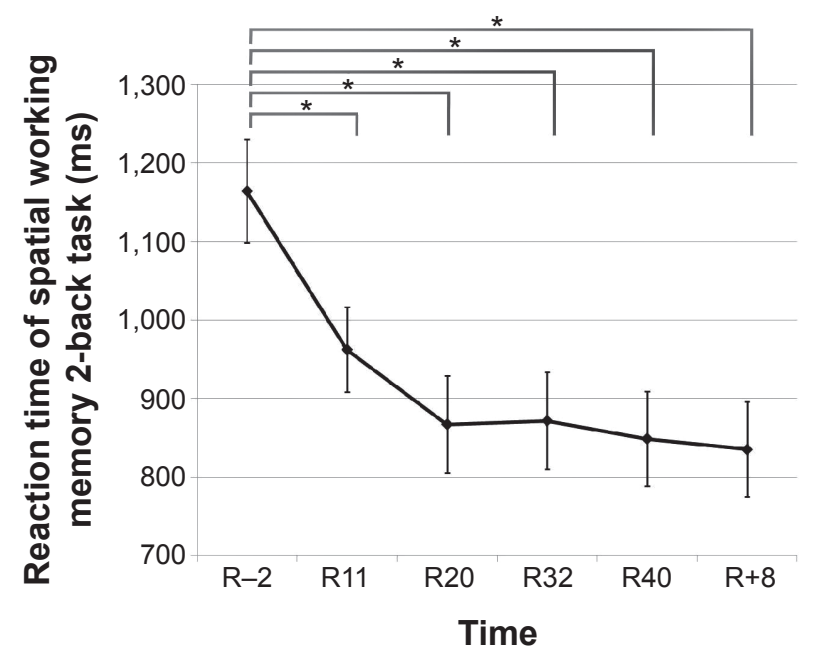

Figure 2 Participants' ACC $(\mathbf{A})$ and $\mathrm{RT}(\mathbf{B})$ of the spatial working memory 2-back task at different time points $(\mathrm{R}-2, \mathrm{RI}, \mathrm{R} 20, \mathrm{R} 32, \mathrm{R} 40$, and $\mathrm{R}+8)$. Notes: Time was expressed in milliseconds; $* P<0.05$.

Abbreviations: ACC, percentage of accurate responses on the 2-back task; HDBR, head-down bed rest; R-2, 2 days before the HDBR; RII, day II of HDBR; R20, day 20 of HDBR; R32, day 32 of HDBR; R40, day 40 of HDBR; R+8, 8 days after the HDBR; RT, mean reaction time on the 2-back task. 
Table 2 Correlations between physiological indices (GSR, HR, and HRV) and ACC, RT of working memory 2-back under HDBR conditions at different time points (R-2, RII, R20, R32, R40, and R+8)

\begin{tabular}{|c|c|c|c|c|c|}
\hline \multirow[t]{4}{*}{ Time } & \multirow{4}{*}{$\begin{array}{l}\text { Physiological } \\
\text { indices }\end{array}$} & \multicolumn{4}{|c|}{ Performance of the 2-back task } \\
\hline & & \multicolumn{2}{|l|}{$\mathbf{R I I}$} & \multirow{3}{*}{$\begin{array}{l}\text { R20 } \\
R T(m s) \\
r(P)\end{array}$} & \multirow{3}{*}{$\begin{array}{l}\text { R32 } \\
R T(\mathrm{~ms}) \\
r(P)\end{array}$} \\
\hline & & $\operatorname{ACC}(\%)$ & RT (ms) & & \\
\hline & & $r(P)$ & $r(P)$ & & \\
\hline \multirow[t]{2}{*}{ RII } & GSR ( $\mu \mathrm{hmo})$ & $-0.528(0.043)^{*}$ & & & \\
\hline & $\mathrm{LF} / \mathrm{HF}$ & & $0.515(0.049)^{*}$ & & \\
\hline R20 & $\mathrm{LF} / \mathrm{HF}$ & & & $0.561(0.030)^{*}$ & \\
\hline R32 & $\mathrm{LF} / \mathrm{HF}$ & & & & $0.547(0.035)^{*}$ \\
\hline
\end{tabular}

Notes: $* P<0.05$. Only the significant correlation results for which the physiological and cognitive responses were consistent (recording on the same day) were reported. Abbreviations: ACC, percentage of accurate responses on the 2-back task; GSR, galvanic skin response; HDBR, head-down bed rest; HF, high frequency; HR, heart rate; LF, low frequency; R-2, 2 days before the HDBR; RII, day II of HDBR; R20, day 20 of HDBR; R32, day 32 of HDBR; R40, day 40 of HDBR; R+8, 8 days after the HDBR; $\mathrm{RT}$, mean reaction time on the 2-back task.

HDBR, there was a correlation between participants' physiological activities and their cognitive performance (ACC and RT of working memory). As time went on, there were significantly positive correlations between the LF/HF and RT of the 2-back task on R20 ( $r=0.561, P=0.030)$ and R32 ( $r=0.547, P=0.035$ ), which suggests that the correlation of physiology and the behavior response under HDBR condition still existed at the two-thirds point of the whole experiment (R32 was the two-thirds of the 45-day HDBR).

\section{Discussion}

The present study had a hypothesis that prolonged bed rest had a detrimental effect on individual physiology. As expected, the results showed that compared with $\mathrm{R}-2$, the participants' GSR increased significantly and the HF and LF decreased significantly after the HDBR $(\mathrm{R}+8)$. This result was in accordance with the perspective of Hirayanagi et al who thought that the bed rest model of prolonged weightlessness was associated with a lack of aerobic physical activity and a decrease in cardiac vagal tone ${ }^{15}$ Moreover, there were positive correlations between the participants' RT of the working memory 2-back task and the LF/HF during the HDBR (R11, R20, and R32). This result was consistent with our second hypothesis that the relation of individual physiology and working memory under HDBR was covariation. This relationship may indicate that while the curve of working memory performance looks like the practice effect of cognition (increased ACC and decreased RT), it actually reflected the detrimental effect of HDBR on working memory ability. The reason for the curve of better performance was the compensatory effect or the sacrifice of the physiological index (decreased LF/HF).

The main purpose of the present study was to investigate whether and to what extent physiology and cognition would be impacted by microgravity analogs, such as
HDBR. As described above, our results showed that the prolonged bed rest did impact individual physiology. It is commonly known that the HDBR simulates microgravity and space flights by physical inactivity, which directly points to the cardiovascular system. ${ }^{6}$ It was not surprising that we found GSR and HRV (HF and LF) variations in our study. On the one hand, GSR measured the electrical resistance of the skin. A transient increase in skin conductance was proportional to sweat secretion. When an individual was under stress environments (the HDBR could be seen as a stress environment), sweat-glands were activated, which increased skin conductance. ${ }^{26}$ The sweat glands were also controlled by the sympathetic nervous system, so the skin conductance acted as an indicator of sympathetic activation due to the stress reaction. On the other hand, like the findings of Fraser et al the HRV (especially the HF) reflected the activity of the vagus nerve in the autonomic nervous system (also called the parasympathetic nervous system) under long-duration International Space Station conditions. ${ }^{27}$ The variations of GSR and HRV (LF and HF) in our study exactly reflected the effect of HDBR on individuals' cardiovascular system, which respectively performed on the sympathetic nervous system and parasympathetic nervous system.

Based on the effect of HDBR on physiology, we would further discuss the variations of physiology under HDBR from the perspective of analog and real space environment comparison.

According to the study of Hockey, long-duration stress environments (such as the International Space Station) had an effect on individuals, causing a fluctuation wave called the "third-quarter phenomenon" ${ }^{28}$ Specifically, individuals in the stress environment would experience anxiety in the prophase, boredom in the metaphase, and excitation in the terminal phase process. The GSR results of the present study 
exactly fit the phenomenon as the GSR decreased as soon as the participants went into HDBR (R11) and then maintained low GSR values until the end of the HDBR (R40); the GSR strengthened on R40 and after the HDBR $(R+8)$ but did not significantly change or return to baseline $(\mathrm{R}-2)$. This result showed that R40 turned out to be an outstanding time point for adapting the long-duration HDBR, which accorded with the third-quarter phenomenon. These results demonstrated that sympathetic neural responses to stress are unaltered by simulated microgravity, just as in the study of Hughson et al. ${ }^{3}$ "The HDBR was a suitable method to mimic physiological variations under weightlessness".

The fluctuation of working memory seen in the 2-back task performance (RT) was in accordance with the physiological index (LF/HF) during HDBR (R11, R20, and R32). This could be interpreted by the findings of Penedo and Dahn that cognition was connected to physiology under stress environment. ${ }^{29}$ The variations of working memory performance may be an outcome of the practice effect on cognitive tasks, since the RT decreased after the individual went into HDBR and continued to decrease after the HDBR. This result was consistent with the study of Kelly et $\mathrm{al}^{30}$ and Zhao et al. ${ }^{31}$ However, the curve of ACC before, during, and after the HDBR could not be interpreted by the practice effect since the ACC after the HDBR decreased from the former time point (R40) and did not have significant increases over that of before the HDBR $(\mathrm{R}-2)$. Furthermore, the decreases of RT were related to the decreases of $\mathrm{LF} / \mathrm{HF}$. This indicated that during HDBR, the decreased LF/HF of participants was used to conduct better cognitive manipulation. In other words, under HDBR, participants adjusted their physiological activity in order to cope with stressors successfully. ${ }^{32}$ Thus, the correlation of task performance and physical response could be interpreted from the perspective of adaptability ${ }^{26}$ and actually, was a compensatory effect. ${ }^{2}$

Above all, the effects of prolonged bed rest on individual physiology and cognition may be detrimental, which mainly manifested as the variations of physiological indices. Physiological indices, such as the GSR and HRV, were sensitive to the variations of the HDBR. Moreover, the results indicated that the prolonged bed rest had a harmful effect on individuals' working memory, though the compensatory effect of LF/ HF. The potential for physiological and cognitive problem development in crews during or after long-duration International Space Station missions requires that consideration be given to prevention and treatment. The increased risk of physiological and cognition problems we observed in simulated microgravity may also be adapted to other highly isolated, confined, physically inactive, and otherwise extreme environments.

\section{Acknowledgments}

This work was funded by the Main Test Technique Research Program of China (grant number 2011CB711000), the "973" project (grant numbers 2011CB711001 and 2011CB505101), and by Shangshan funding. The authors would like to express their gratitude for the support of these projects.

\section{Disclosure}

The authors report no conflicts of interest in this work.

\section{References}

1. Crucian B, Simpson RJ, Mehta S, et al. Terrestrial stress analogs for spaceflight associated immune system dysregulation. Brain Behav Immun. 2014;39:23-32.

2. Lipnicki DM, Gunga HC. Physical inactivity and cognitive functioning: results from bed rest studies. Eur J Appl Physiol. 2009;105(1):27-35.

3. Hughson RL, Shoemaker JK, Blaber AP, et al. Cardiovascular regulation during long-duration spaceflights to the International Space Station. J Appl Physiol (1985). 2012;112(5):719-727.

4. Pavy-Le Traon A, Heer M, Narici MV, Rittweger J, Vernikos J. From space to Earth: advances in human physiology from 20 years of bed rest studies (1986-2006). Eur J Appl Physiol. 2007;101(2):143-194.

5. Ritsher JB, Kanas NA, Ihle EC, Saylor SA. Psychological adaptation and salutogenesis in space: Lessons from a series of studies. Acta Astronaut. 2007;60(4-7):336-340.

6. Coupé M, Fortrat JO, Larina I, Gauquelin-Koch G, Gharib C, Custaud MA. Cardiovascular deconditioning: From autonomic nervous system to microvascular dysfunctions. Respir Physiol Neurobiol. 2009; 169 Suppl 1:S10-S12.

7. Aubert AE, Verheyden B, d'Y dewalle C, Beckers F, Van den Bergh O. Effects of mental stress on autonomic cardiac modulation during weightlessness. Am J Physiol Heart Circ Physiol. 2010;298(1):H202-H209.

8. Lipnicki DM, Gunga H-C, Belavý DL, Felsenberg D. Bed Rest and Cognition: Effects on Executive Functioning and Reaction Time. Aviat Space Envir Md. 2009;80(12):1018-1024.

9. Ishizaki Y, Fukuoka H, Tanaka H, et al. Executive function on the 16-day of bed rest in young healthy men. Acta Astronaut. 2009;64(9-10): 864-868.

10. Seaton KA, Slack KJ, Sipes WA, Bowie KE. Cognitive functioning in long-duration head-down bed rest. Aviat Space Environ Med. 2009; 80(5 Suppl):A62-A65.

11. Shehab RL, Schlegel RE, Schiflett SG, Eddy DR. The NASA Performance Assessment Workstation: cognitive performance during headdown bed rest. Acta Astronaut. 1998;43(3-6):223-233.

12. Crucian BE, Stowe RP, Mehta SK, et al. Immune status, latent viral reactivation, and stress during long-duration head-down bed rest. Aviat Space Environ Med. 2009;80(5 Suppl):A37-A44.

13. Erland A, Svensson I, Wilson GF. Psychological and psychophysiological models of pilot performance for systems development and mission evaluation. Int J Aviat Psychol. 2002;12(1):95-110.

14. Thayer JF, Hansen AL, Saus-Rose E, Johnsen BH. Heart rate variability, prefrontal neural function, and cognitive performance: the neurovisceral integration perspective on self-regulation, adaptation, and health. Ann Behav Med. 2009;37(2):141-153.

15. Hirayanagi K, Iwase S, Kamiya A, Sasaki T, Mano T, Yajima K. Functional changes in autonomic nervous system and baroreceptor reflex induced by 14 days of 6 degrees head-down bed rest. Eur J Appl Physiol. 2004;92(1-2):160-167. 
16. Esposito M, Verrotti A, Gimigliano F, et al. Motor coordination impairment and migraine in children: a new comorbidity? Eur J Pediatr. 2012;171(11):1599-1604.

17. Esposito M, Antinolfi L, Gallai B, et al. Executive dysfunction in children affected by obstructive sleep apnea syndrome: an observational study. Neuropsychiatr Dis Treat. 2013;9:1087-1094.

18. Esposito M, Carotenuto M. Borderline intellectual functioning and sleep: the role of cyclic alternating pattern. Neurosci Lett. 2010;485(2):89-93.

19. Esposito M, Carotenuto M. Intellectual disabilities and power spectra analysis during sleep: a new perspective on borderline intellectual functioning. J Intellect Disabil Res. 2014;58(5):421-429.

20. Esposito M, Gallai B, Parisi L, et al. Visuomotor competencies and primary monosymptomatic nocturnal enuresis in prepubertal aged children. Neuropsychiatr Dis Treat. 2013;9:921-926.

21. Esposito M, Pascotto A, Gallai B, et al. Can headache impair intellectual abilities in children? An observational study. Neuropsychiatr Dis Treat. 2012;8:509-513.

22. Liu Q, Zhou R, Chen S, Tan C. Effects of head-down bed rest on the executive functions and emotional response. PLoS One. 2012;7(12):e52160.

23. Chen YN, Mitra S, Schlaghecken F. Sub-processes of working memory in the N-back task: an investigation using ERPs. Clin Neurophysiol. 2008;119(7):1546-1559.

24. Chen S, Zhou R, Xiu L, Chen S, Chen X, Tan C. Effects of 45-day -6 ${ }^{\circ}$ head-down bed rest on the time-based prospective memory. Acta Astronaut. 2013;84:81-87.
25. Sztajzel J. Heart rate variability: a noninvasive electrocardiographic method to measure the autonomic nervous system. Swiss Med Wkly. 2004;134(35-36):514-522.

26. Wieser M, Buetler L, Vallery H, et al. Quantification of clinical scores through physiological recordings in low-responsive patients: a feasibility study. J Neuroeng Rehabil. 2012;9:30-41.

27. Fraser KS, Greaves DK, Shoemaker JK, Blaber AP, Hughson RL. Heart rate and daily physical activity with long-duration habitation of the International Space Station. Aviat Space Environ Med. 2012;83(6): 577-584.

28. Hockey GR. Compensatory control in the regulation of human performance under stress and high workload; a cognitive-energetical framework. Biol Psychol. 1997;45(1-3):73-93.

29. Penedo FJ, Dahn JR. Exercise and well-being: a review of mental and physical health benefits associated with physical activity. Curr Opin Psychiatry. 2005;18(2):189-193.

30. Kelly TH, Hienz RD, Zarcone TJ, Wurster RM, Brady JV. Crewmember performance before, during, and after spaceflight. J Exp Anal Behav. 2005;84(2):227-241.

31. Zhao X, Wang Y, Zhou R, Wang L, Tan C. The influence on individual working memory during 15 days $-6^{\circ}$ head-down bed rest. Acta Astronaut. 2011;69:969-974.

32. Plessow F, Fischer R, Kirschbaum C, Goschke T. Inflexibly focused under stress: acute psychosocial stress increases shielding of action goals at the expense of reduced cognitive flexibility with increasing time lag to the stressor. J Cogn Neurosci. 2011;23(11):3218-3227.
Neuropsychiatric Disease and Treatment

\section{Publish your work in this journal}

Neuropsychiatric Disease and Treatment is an international, peerreviewed journal of clinical therapeutics and pharmacology focusing on concise rapid reporting of clinical or pre-clinical studies on a range of neuropsychiatric and neurological disorders. This journal is indexed on PubMed Central, the 'PsycINFO' database and CAS,

\section{Dovepress}

and is the official journal of The International Neuropsychiatric Association (INA). The manuscript management system is completely online and includes a very quick and fair peer-review system, which is all easy to use. Visit http://www.dovepress.com/testimonials.php to read real quotes from published authors. 